\title{
RECURRENCE-TIME MOMENTS IN RANDOM WALKS
}

\author{
J. L. HOdges, JR. ANd M. ROSENBlatt
}

1. Introduction. Consider an irreducible time-homogeneous Markov chain with discrete time. The recurrence-time moments of the states of such stochastic processes are studied. We point out that if the recurrence time of one state has its first $k$ moments finite, then the recurrence times of all the other states have their first $k$ moments finite. We then specialize and investigate the recurrencetime moments of random walks. The main result of the paper consists of exhibiting random walks whose first $k-1$ recurrence-time moments exist and whose higher moments are infinite, for $k=1,2, \ldots$. A comparison theorem is derived that permits the moment properties of recurrence times of a large class of random walks to be determined.

2. Preliminary considerations. We begin with the following:

DEFinition. By the index of the random variable $X$, denoted by $I(X)$, we shall mean the largest integer $k$ such that $E\left(X^{k}\right)<\infty$. If all moments of $X$ are finite, we write $I(X)=\infty$. Clearly $I(X) \geq 0$. We shall consider only nonnegative random variables.

Lemma $1 .^{1}$ (a) If $X_{1}, \ldots, X_{n}$ are independent random variables, then

$$
I\left(X_{1}+\cdots+X_{n}\right)=\min \left\{I\left(X_{1}\right), \cdots, I\left(X_{n}\right)\right\} .
$$

(b) If $X$ has the geometric distribution

$$
P(X=n)=p(1-p)^{n-1} \quad(p>0),
$$

then $I(X)=\infty$.

(c) If $N, X_{1}, X_{2}, \ldots$ are independent random variables, $N$ is positive integer valued, $X_{1}, X_{2}, \ldots$ are identically distributed, and then

\footnotetext{
${ }^{1}$ The proof of these simple facts is omitted.

Received April 28, 1952. This work has been carried out under ONR contract.

Pacific J. Math. 3 (1953), 127- 136
} 


$$
I\left(X_{1}+\cdots+X_{N}\right)=\min \left\{I(N), I\left(X_{1}\right)\right\}
$$

We adopt the terminology and notation for Markov chains used by Feller [1, Chap. 15]. Let $E_{1}, E_{2}, \ldots$ be a denumerable set of states. We assume the chain is irreducible; i.e., for every pair $E_{i}, E_{j}$ there exists $n(i, j)$ such that transition from $E_{i}$ to $E_{j}$ in $n(i, j)$ steps has positive probability. Let $R_{i}$ be the random number of steps for first return to $E_{i}$, and let $R_{i j}$ be the random number of steps for first passage from $E_{i}$ to $E_{j}$, starting at $E_{i}$. It is well known [1] that if, for any $j, P\left(R_{j}<\infty\right)=1$, then this holds true for all $j$, and we shall assume this to be the case; i.e., our process is assumed to be recurrent.

LEMmA $2 .^{2}$ (a) $I\left(R_{i}\right)$ has a constant value, say $I(C)$, for all $i=1,2, \ldots$.

(b) For every pair $i \neq j$,

$$
I\left(R_{i}\right)=\min \left\{I\left(R_{i j}\right), I\left(R_{j i}\right)\right\}
$$

This lemma is related to the well-known result that in a recurrent irreducible time-homogeneous Markov chain, if the expected time for first return to any state is finite, then the same holds for all states. In the language of [1, Chap. 15], if any state is null, then every state is null. The lemma extends this result from first moment to arbitrary moments.

Examples of Markov chains of index $k$ are to be found in a class of Markov chains considered by Feller [1]. Let $X(n)$ be the Markov chain, and let

$$
\begin{aligned}
& p_{j}=P\left\{X(n)=E_{j+1} \mid X(n-1)=E_{j}\right\}, \\
& q_{j}=1-p_{j}=P\left\{X(n)=E_{1} \mid X(n-1)=E_{j}\right\}, j=1,2, \cdots .
\end{aligned}
$$

Then

$$
P\left(R_{1}=n\right)=\left(1-p_{n}\right) \prod_{i=1}^{n-1} p_{i}=\prod_{i=1}^{n-1} p_{i}-\prod_{i=1}^{n} p_{i}
$$

We obviously obtain a Markov chain with $E\left(R_{1}^{k}\right)<\infty, E\left(R_{1}^{k+i}\right)=\infty$, where $i \geq 1$, if the $p_{i}$ are such that

$$
\prod_{1}^{n-1} p_{i}-\prod_{1}^{n} p_{i}=\frac{C}{n^{k+1+\epsilon}} \quad(0<\epsilon<1)
$$

2 After obtaining this result we learned that it had been obtained earlier by K. L. Chung and by R. N. Snow, in a more general form (unpublished). We omit the proof. 
where

$$
C \sum_{1}^{\infty} \frac{1}{n^{k+1+\epsilon}}=1 \text {. }
$$

3. Comparison theorem for random walks. We shall now specialize from Markov chains to random walks. There is no loss of generality in taking the states of the walk to be consecutive integers, and we shall let $p_{i}$ denote the probability of transition from $i$ to $i+1$. To ensure irreducibility, we shall require $0<p_{i}<1$ for all interior states. To apply Lemma 2, we must have the walk recurrent, so we shall assume that the boundaries, if any, are reflecting, and that (if the walk is unbounded in one or both directions) the probability of escape to infinity is 0 . Denote the walk by $W$ and its index by $I(W)$.

THEOREM 1. (a) $I\left(R_{i j}\right)$ has a constant value, say $I^{R}$, for all $i>j$, and a constant value, say $I^{L}$, for all $i<j$.

$$
\text { (b) Further, } I(W)=\min \left\{I^{L}, I^{R}\right\} \text {. }
$$

Proof. We shall first show that $I\left(R_{i+1, i}\right)=I\left(R_{i, i-1}\right)$. Consider a walk starting at $i$ and indefinitely prolonged. It is certain eventually to reach $i-1$; let $M$ denote the number of times the walk is at $i$, including the start, before reaching $i-1 ; M$ has the distribution

$$
P(M=m)=\left(1-p_{i}\right) p_{i}^{m-1}
$$

and given $M=m$, the walk consists of $m-1$ steps from $i$ to $i+1, m-1$ first passages from $i+1$ to $i$ (denote these by $R_{i+1, i}^{(1)}, \cdots, R_{i+1, i}^{(m-1)}$ ), and a terminal step from $i$ to $i-1$. Thus

$$
R_{i, i-1}=M+R_{i+1, i}^{(1)}+\cdots+R_{i+1, i}^{(M)}
$$

Apply Lemma 1 (b), (c), (a).

To complete the proof of (a), represent a walk from $i$ to $j, i>j$, as the sum

$$
R_{i, j}=R_{i, i-1}+R_{i-1, i-2},+\cdots+R_{j+1, j},
$$

and apply Lemma 1 (a). As for (b), this follows from Lemma 2 (b).

It is clear that in a walk over finitely many states, the index is $\infty$. (See [1, problem 8, p. 345].) Similarly, passages away from a reflecting boundary 
have moments of all orders. The interest attaches to passages away from an unbounded side, and by virtue of Theorem 1 (b) we may consider the two sides separately. We may therefore, without loss of generality, consider only passages to the left in semi-infinite walks unbounded on the right, and we may take the states to be nonnegative integers. We now approach the following general question: given the sequence of transition probabilities $p_{1}, p_{2}, \ldots$ of such a walk, to determine the index of first passages from right to left.

This question is somewhat analogous to the question of determining the minimum integer $k$ such that the series $\sum X_{i}^{k}$ is convergent. As in the convergence of series, all that matters is the performance of $\left\{p_{i}\right\}$ in the tail-any finite number of terms may be changed or suppressed without altering the result. We also have a "comparison" theorem.

THEOREM 2. Consider two random walks, W and $V$, with transition probabilities $\left\{p_{i}\right\}$ and $\left\{q_{i}\right\}$, respectively. If $p_{i} \leq q_{i}$ for every $i=1,2, \cdots$, then $I(W) \geq I(V)$.

Proof. We may assume that the walks begin at the same state. Refer both walks to the same infinite process $X_{1}, X_{2}, \ldots$, where $X_{1}, X_{2}, \ldots$ are mutually independent random variables, each uniformly distributed over $(0,1)$. If the walk $W$ is at state $j$ for trial $i$, it proceeds to the right if and only if $X_{i}<p_{j}$, and similarly for walk $V$, which proceeds to the right if and only if $X_{i}<q_{j}$.

Consider $X_{1}, X_{2}, \ldots$ fixed at observed values $x_{1}, x_{2}, \ldots$. Observe that $W$ and $V$ always differ by an even number and that this difference changes by either 0 or 2 on each step. We shall show that walk $W$ can never be to the right walk $V$. If it were, there would have to be a first time on which this occurred, and on the preceding time, say trial $i$, the walks would coincide, say at state $j$. But $p_{j} \leq q_{j}$, so that $x_{i}<p_{j}$ and $x_{i} \geq q_{j}$ are contradictory.

As an application, we remark: if $\lim$ sup $p_{i}<1 / 2$, then the index is $\infty$; and if $\lim \inf p_{i}>1 / 2$, when the walk is nonrecurrent. This fact follows from comparison with the classical walk with constant transition probability $p$.

The fact just cited indicates that the interest will lie in those sequences $\left\{p_{i}\right\}$ for which $\lim \sup p_{i} \geq 1 / 2$ and $\lim$ inf $p_{i} \leq 1 / 2$. We shall in the next section investigate a class of walks in which $\lim p_{i}=1 / 2$, and these will serve, together with the comparison theorem, to handle a large class of problems.

4. A class of random walks. We now consider a class of random walks which are related to the ordinary unbiased coin tossing. The random walk has as its possible states the integers $r, r+1, r+2, \cdots$. Let 


$$
\begin{aligned}
p_{k} & =P\{X(n)=k+1 \mid X(n-1)=k\}, \\
1-p_{k} & =q_{k}=P\{X(n)=k-1 \mid X(n-1)=k\}, \\
q_{k, n} & =P\{\text { going from } k \text { to } r \text { for the first time in } n \text { steps }\} \quad(k>r) .
\end{aligned}
$$

Then

$$
q_{k, n+1}=p_{k} q_{k+1, n}+q_{k} q_{k-1, n} \quad(k>r) .
$$

The state $r$ will be an absorbing barrier. This implies that

$$
q_{r, 0}=1 \text {, while } q_{r, n}=0 \text { if } n>0 \text {. }
$$

We shall set $q_{r-1, n}=0$ for all $n$ as a convenient convention. Note that

$$
\begin{aligned}
& q_{r+1,1}=q_{r+1}, \text { while } q_{k, 1}=0 \text { if } k>r+1 ; \\
& q_{k, 0}=0 \text { if } k>r .
\end{aligned}
$$

Define

$$
G_{k}(s)=\sum_{n=0}^{\infty} q_{k, n} s^{n} \quad(k=r-1, r, r+1, \cdots),
$$

and observe that

$$
G_{r-1}(s)=0, G_{r}(s)=1 \text {. }
$$

Let

$$
p_{k}=\frac{1}{2}\left(1-\frac{r}{k}\right), q_{k}=\frac{1}{2}\left(1+\frac{r}{k}\right) \quad(k=r, r+1, \ldots) .
$$

Note that $r=0$ gives us the ordinary case of unbiased coin tossing.

The generating functions satisfy the following equations:

$$
\begin{aligned}
& G_{k}(s)=p_{k} s G_{k+1}(s)+q_{k} s G_{k-1}(s) \quad(k>r), \\
& G_{r}(s)=p_{r} s G_{r+1}(s)+q_{r} s G_{r-1}(s)+1=q_{r} s G_{r-1}(s)+1 .
\end{aligned}
$$

Let 


$$
\begin{aligned}
& G(t, s)=\sum_{k=r}^{\infty} G_{k}(s) t^{k}= \\
& \quad \frac{s}{2}\left[\frac{G(t, s)-t^{r}}{t}+t G(t, s)\right]+t^{r}+\frac{s r}{2}\left[\int_{0}^{t} G(t, s) d t-\int_{0}^{t} \frac{G(t, s)-t^{r}}{t^{2}} d t\right] .
\end{aligned}
$$

We now differentiate with respect to $t(0<t<1)$, use $[1-(s / 2)(1 / t+t)]^{r+1}$ as an integrating factor, and integrate the differential equation between the limits $t$ and $\sigma=\left[1-\left(1-s^{2}\right)^{1 / 2}\right] / s$, where $0<t<\sigma$. We then obtain

$$
\begin{aligned}
-G(t, s)[1- & \left.\frac{s}{2}\left(\frac{1}{t}+t\right)\right]^{r+1} \\
& =\int_{t}^{\sigma}\left[\frac{s \tau^{r-2}}{2}+r \tau^{r-1}\right]\left[1-\frac{s}{2}\left(\frac{1}{\tau}+\tau\right)\right]^{r} d \tau .
\end{aligned}
$$

Let ${ }_{c} \mu_{k}$ denote the $c$ th factorial moment of the first passage time from $k$ to $r$. It is easily seen that the ${ }_{c} \mu_{k}$ (c fixed), if they are finite, cannot increase with $k$ more than exponentially. Therefore, if ${ }_{c} \mu_{k}<\infty$ for some positive $c$ and some $k>r$, then

$$
\sum_{k=r}^{\infty} c^{\mu_{k}} t^{k-c}
$$

is convergent for sufficiently small $t$. Therefore

$$
\lim _{s \rightarrow 1_{-}^{-}} \frac{d^{c}}{d s^{c}} G(t, s)=\sum_{k=r}^{\infty} c^{\mu_{k}} t^{k-c}
$$

exists for sufficiently small $t$ if and only if ${ }_{c} \mu_{k}<\infty$ for some $k>r$ (and hence for all $k>r$ ).

It is now easily shown that the first $r$ derivatives of the integral on the right of (1) with respect to $s$ are bounded in the closed interval $(t, 1)$, and hence have finite limits as $s \rightarrow 1-$. The $(r+1)$ st derivative, however, contains a term with the factor $d \sigma / d s$, which diverges as $s \rightarrow 1-$.

The same techniques can be used to prove the following result: 
THEO REM 3. If one has a one-sided random walk with

$$
p_{k}=\frac{1}{2}\left(1-\frac{\alpha}{k}\right), q_{k}=\frac{1}{2}\left(1+\frac{\alpha}{k}\right)
$$

$\alpha>0(k=\alpha, \alpha+1, \ldots)$ then ${ }_{r} \mu_{k}<\infty$ if $r-1 / 2<\alpha \leq r+1 / 2$ and $k>\alpha$, while ${ }_{c} \mu_{k}=\infty$ for $c>r$ and $k>\alpha$.

We shall now consider walks with $p_{n}>1 / 2$. It is clear from Theorem 2 that for such walks the index is 0 . The interest now attaches to the question of whether first passages are certain to occur.

Lemm A $3 .{ }^{3}$ Consider a random walk over the states $0,1,2, \ldots$. Assume $p_{k} \geq \epsilon$ for some $\epsilon>0$. The walk is recurrent if and only if the series

$$
\sum_{k=1}^{\infty} \frac{q_{1} q_{2} \cdots q_{k}}{p_{1} p_{2} \cdots p_{k}}
$$

is divergent.

Proof. Consider first the finite walk over $0,1,2, \ldots, n$, and let $P_{k}^{(n)}$ denote the probability that the walk, starting at $k$, reaches $n$ before reaching 0 . Clearly

$$
P_{0}^{(n)}=0, P_{n}^{(n)}=1
$$

and

$$
P_{k}^{(n)}=p_{k} P_{k+1}^{(n)}+q_{k} P_{k=1}^{(n)} \quad(k=1,2, \ldots, n-1) .
$$

We may solve for $P_{2}^{(n)}, P_{3}^{(n)}, \ldots, P_{n-1}^{(n)}$ in terms of $P_{1}^{(n)}$, getting

$$
q_{k}\left(P_{k}^{(n)}-P_{k-1}^{(n)}\right)=p_{k}\left(P_{k+1}^{(n)}-P_{k}^{(n)}\right) \quad(k=1,2, \cdots, n-1),
$$

and hence

$$
P_{k}^{(n)}=\left(1+b_{1}+b_{2}+\cdots+b_{k-1}\right) P_{1}^{(n)}
$$

where $b_{j}=\left(q_{1} q_{2} \cdots q_{j}\right) /\left(p_{1} p_{2} \cdots p_{j}\right)$. Now suppose $\sum_{j=1}^{\infty} b_{j}=\infty$. Since

${ }^{3}$ We were informed by the referee that this lemma and the following theorem have also been obtained by T. E. Harris. 


$$
P_{1}^{(n)}=P_{n-1}^{(n)} /\left(1+\sum_{k=1}^{n-1} b_{k}\right) \leq 1 /\left(1+\sum_{k=1}^{n-1} b_{k}\right),
$$

we must have $P_{1}^{(n)} \longrightarrow 0$ as $n \rightarrow \infty$. We conclude that the walk is recurrent.

Conversely, suppose $\sum_{k=1}^{\infty} b_{k}<\infty$. Then $\sum_{k=1}^{\infty} b_{k}=b_{0}$, where $b_{0}$ is some positive number. Using the fact that

$$
P_{n-1}^{(n)}=p_{n-1}+q_{n-1} P_{n-2}^{(n)}
$$

we have

$$
\left(1+\sum_{k=1}^{n-1} b_{k}\right) P_{1}^{(n)}=p_{n-1}+q_{n-1}\left(1+\sum_{k=1}^{n-2} b_{k}\right) P_{1}^{(n)},
$$

and therefore

$$
P_{1}^{(n)}=1 /\left[1+\sum_{k=1}^{n-2} b_{k}+b_{n-1} / p_{n-1}\right]
$$

Thus

$$
\lim _{n \rightarrow \infty} P_{1}^{(n)}=1 / b_{0}>0
$$

whence the walk is nonrecurrent.

As an illustration, consider a random walk over the nonnegative integers with reflecting barrier 0 and

$$
p_{k}=\frac{1}{2}\left(1+\frac{1}{\beta k}\right), \quad(k=1,2, \ldots ; \beta>1) .
$$

THEOREM 4. The walk is recurrent if and only if $\beta \geq 2$.

Proof. Let

$P_{k}=P\{$ walk never reaches 0, given that it starts at $k\} \quad(k=1,2, \ldots)$.

We have 
$P_{1}=\frac{1}{2}\left(1+\frac{1}{\beta}\right) P_{2}, P_{k}=\frac{1}{2}\left(1-\frac{1}{\beta k}\right) P_{k-1}+\frac{1}{2}\left(1+\frac{1}{\beta k}\right) P_{k+1} \quad(k=2,3, \cdots)$.

Rewriting, we have

$P_{2}-P_{1}=\frac{\beta-1}{\beta+1} P_{1}, P_{k+1}-P_{k}=\frac{\beta k-1}{\beta k+1}\left(P_{k}-P_{k-1}\right) \quad(k=2,3, \cdots)$.

Thus

$$
P_{k}=P_{1}\left\{1+\frac{\beta-1}{\beta+1}+\frac{\beta-1}{\beta+1} \cdot \frac{2 \beta-1}{2 \beta+1}+\cdots+\left(\frac{\beta-1}{\beta+1} \cdots \frac{(k-1) \beta-1}{(k-1) \beta+1}\right)\right\} .
$$

Recalling the preceding lemma, we see that the recurrence of the walk is equivalent to the divergence of

$$
1+\frac{\beta-1}{\beta+1}+\frac{\beta-1}{\beta+1} \cdot \frac{2 \beta-1}{2 \beta+1}+\frac{\beta-1}{\beta+1} \cdot \frac{2 \beta-1}{2 \beta+1} \cdot \frac{3 \beta-1}{3 \beta+1}+\cdots=f(\beta) .
$$

We easily see that

$$
f(2)=1+\frac{1}{3}+\frac{1}{5}+\cdots=\infty,
$$

so the walk is recurrent when $\beta=2$. That it is recurrent for $\beta>2$ then follows from the lemma. The series $f(\beta)(1<\beta<2)$, may be shown to be convergent as follows. For $1<\beta<2$,

$$
\begin{aligned}
f(\beta) & =1+\frac{\beta-1}{\beta+1}+\frac{\beta-1}{2 \beta+1}\left(1-\frac{2-\beta}{\beta+1}\right)+\frac{\beta-1}{3 \beta+1}\left(1-\frac{2-\beta}{\beta+1}\right)\left(1-\frac{2-\beta}{2 \beta+1}\right)+\cdots \\
& <1+\frac{1}{2}+\frac{1}{3}\left(1-\frac{2-\beta}{3}\right)+\frac{1}{4}\left(1-\frac{2-\beta}{3}\right)\left(1-\frac{2-\beta}{5}\right)+\cdots
\end{aligned}
$$

But

$$
\begin{gathered}
\left(1-\frac{2-\beta}{3}\right)\left(1-\frac{2-\beta}{5}\right) \cdots\left(1-\frac{2-\beta}{2 k+1}\right)<e^{-(2-\beta)(1 / 3+1 / 5+\cdots+1 / 2 k+1)} \\
<e^{[-(2-\beta) / 4] \log (2 k+1)}=(2 k+1)^{-(2-\beta) / 4} .
\end{gathered}
$$

Thus 


$$
f(\beta)<1+\frac{1}{2}+\frac{1}{3} \frac{1}{3^{(2-\beta) / 4}}+\frac{1}{4} \frac{1}{5^{(2-\beta) / 4}}+\cdots<\infty .
$$

The preceding class of examples of random walks together with the comparison theorem (Theorem 2) now permit us to determine the moment properties of a large class of random walks. For example, the one-sided walks with

$$
p_{n}=\frac{1}{2}\left(1+\frac{\alpha}{n^{\beta}}\right) \quad(n=1,2, \cdots)
$$

have the same moment index as the walk with $p_{n}=1 / 2$ if $\alpha>0, \beta>1$, while if $\beta<1, \alpha<0$ the index is infinity (all moments exist). For $\alpha>0$, the walk will be certain to return if $\beta<1$, but not if $\beta>1$.

\section{REFERENCE}

1. W. Feller, An introduction to probability theory and its applications, Wiley, New York, 1950.

University of Chicago 